\title{
NOUVEL ESSAI DE CLASSIFICATION DES FILAIRES
}

\section{(SUPERFAMILLE DES FILARIOÏDEA)}

\author{
Par Alain-G. CHABAUD et Marie-Thérèse CHOQUET
}

La classification des Filaires pose un des problèmes systématiques les plus difficiles qui puissent se trouver en Helminthologie. Il s'agit en effet de Nématodes très profondément modifiés par leur biologie ; leur adaptation à la vie tissulaire a amené des réductions d'organes et des convergences tellement marquées, qu'il est souvent impossible de préciser l'origine, et la filiation approximative des différents genres.

S'il est impossible d'établir une phylogénie cohérente, et cela d'autant plus que le groupe paraît essentiellement polyphylétique, nous croyons pourtant qu'il est possible de schématiser grossièrement les différentes étapes qui conduisent des formes primitives, proches des Spiruroïdes intestinaux, aux formes les plus étroitement spécialisées à leur milieu.

\section{I. - Evolution des caractères au cours de l'adaptation à la vie tissulaire}

Il est certain que l'oviparité est plus ancienne que la viviparité. Les Filaires qui pondent des œufs à coque épaisse et dont les larves sont armées d'épines sont évidemment plus proches des Spirurides intestinaux que les Filaires qui émettent des microfilaires inermes circulant dans le sang. Il y a naturellement de nombreuses formes de passage, et il est important de rapprocher les Filaires vivipares, qui ont des larves spinulées, des formes ovipares ; et, inversement, de rapprocher les Filaires, qui pondent des larves inermes entourées d'une fine membrane, des espèces vivipares.

Cette première coupure biologique coïncide d'ailleurs presque toujours avec les éléments apportés par la morphologie.

La spécialisation à la vie tissulaire s'accompagne en effet de réductions de tous les organes, à l'exception de l'appareil génital ; mais

AnN. de Parasitologie, T. XXVIII, N ${ }^{\circ} 3 .-1953$. 
ces réductions paraissent suivre une marche plus ou moins progressive et certains organes traduire plus fidèlement les différentes étapes de la spécialisation. Il semble donc indispensable d'établir une hiérarchie dans l'étude de ces régressions.

Par ordre d'importance, les éléments les plus intéressants nous paraissent être les suivants :

1. Le nombre et la situation des papilles céphaliques ; ils paraissent devoir donner, comme chez les Spiruroïdes intestinaux, des renseignements d'une très grande valeur, mais malheureusement ils sont si mal connus chez la plupart des espèces, qu'il est actuellement presque toujours impossible de s'en servir.

2. L'ornementation cuticulaire péribuccale, mieux connue, donne au contraire des éléments valables pour une classification de base, et ces éléments coïncident d'ailleurs presque toujours avec les caractères larvaires.

3. Presque toutes les Filaires présentent un déplacement de la vulve vers la région antérieure, et les quelques rares formes qui ont conservé une vulve postérieure paraissent primitives.

4. De la même façon, l'anus tend à se placer dans une situation de plus en plus postérieure ; ce caractère, généralement négligé dans les classifications générales, nous paraît devoir fournir des éléments de diagnose supra-génériques valables.

5. La régression de la capsule buccale donne des indications importantes, mais souvent difficiles à utiliser, car elle peut atteindre des degrés très différents chez des formes proches les unes des autres.

6. Les caractères donnés par les spicules ont peu d'importance phylogénique, mais on se trouve amené à les utiliser dans les coupures entre sous-familles, car c'est un des éléments les plus faciles à observer et les mieux connus dans l'anatomie des Filaires.

7. La réduction et la simplification de l'œsophage, l'amphidelphie, la disparition des diérides, la perte des ailes caudales, l'atrophie des papilles cloacales sont autant d'éléments qui indiquent une spécialisation de plus en plus marquée ; mais ces évolutions ne se font pas toujours de façon parallèle, et l'on ne peut souvent utiliser ces éléments que pour des diagnoses génériques ou même seulement spécifiques. 


\section{II. - Classifications actuelles des filaires}

Les tableaux dichotomiques actuellement proposés pour la détermination générique des Filaires sont très peu nombreux.

Celui de Yorke et Maplestone, très ancien (1926), n'est plus guère utilisé, car il fait essentiellement appel à des caractères cuticulaires dont on a universellement reconnu le peu de valeur générique.

Celui de Skrjabin et Schikhobalowa (1936), partiellement remis à jour par Lopez-Neyra (1947), a été remanié plus récemment par Skrjabin et Schikhobalowa (1948). Il est malheureusement entièrement rédigé en russe et très difficile à se procurer en France. Cependant, cet important travail s'apparente plus à un fichier par ordre alphabétique qu'à une classification zoologique. Comme le fait remarquer Caballero (1951), on y trouve en effet un certain nombre d'espèces mises depuis longtemps en synonymie ou reconnues appartenir à des genres très différents. Mais, surtout, les coupures entre les familles ou sous-familles sont si rares et si artificielles que les genres les plus divers sont étudiés les uns après les autres, alors que des genres très proches sont complètement dispersés. On s'étonne ainsi de voir l'Hamatospiculum otomelarum Tubangui 1934 (figuré p. 210) si éloigné du Parhamatospiculum cylindrica (Zeder 1803) (figuré p. 511), alors que les genres Paralemdana et Paraprocta sont traités par ordre alphabétique, à la suite de Parafilaria. Une telle confusion nous paraît inévitable lorsque l'on emploie comme base de classification la longueur des spicules.

Au contraire, la classification proposée par Wehr (1935) parait très satisfaisante, car elle se base sur les éléments qui semblent avoir la meilleure valeur phylogénique, et en particulier sur les caractères larvaires et les caractères céphaliques. Les éléments spécialement sensibles aux phénomènes de convergence, tels que l'aspect cuticulaire ou la taille des spicules, ne sont utilisés, au contraire, que pour les séparations génériques ou spécifiques. On obtient ainsi une classification qui, au premier abord, parait d'un emploi plus délicat, mais qui se révèle plus satisfaisante et plus efficace dans les cas difficiles.

Le syștème de Wehr n'a cependant pu être utilisé que par quelques rares spécialistes, car l'auteur a préféré ne pas donner de tables dichotomiques, et l'on ne trouve que 46 genres qui soient classés, alors qu'il en existe actuellement presque le double.

Moins prudents que l'auteur américain, nous avons préféré ici classer tous les genres venus à notre connaissance ; nous nous exposons ainsi à commettre un certain nombre d'erreurs, car les caractè- 
res céphaliques et larvaires de beaucoup de formes sont encore mal précisés, mais l'absence totale de travaux récents et facilement accessibles nous fait croire que la publication de nouveaux tableaux dichotomiques est utile.

\section{III. - Classification proposée}

Nous adopterons à peu près exactement le cadre donné par Wehr, à l'exception cependant de la famille des Dipetalonematidæ. La division en deux sous-familles, dont l'une, les Dipetalonematinæ, groupe plus de la moitié des genres de Filaires connus, paraît en effet d'un emploi difficile et nous semble rapprocher des formes trop différentes les unes des autres.

Le meilleur caractère de base permettant de diviser les Dipetalonematinæ en groupes homogènes nous a paru être la longueur de la queue. C'est un élément qui paraît avoir une certaine valeur phylogénique, car la migration de l'anus en position de plus en plus postérieure s'observe chez les espèces très spécialisées, et l'emploi de ce caractère dans les tableaux dichotomiques ne soulève pas les difficultés auxquelles on pourrait s'attendre d priori. Il est en effet remarquable de constater :

a) que la longueur de la queue est toujours comparable dans les deux sexes d'une même espèce. A de très rares exceptions près, on ne trouve pas de mâle à queue longue, lorsque la femelle a une queue courte (et inversement) ;

b) que, dans les groupes où nous aurons l'occasion de nous servir de cet élément de diagnose, les queues sont tantôt franchement longues, tantôt franchement courtes; il se trouve que dans les cas assez rares, où la queue, de longueur moyenne, laisse place à une hésitation, les espèces ont été éliminées par d'autres caractères dans des groupes distincts. Il nous a fallu seulement écarter du genre Dipetalonema deux espèces, remarquables d'ailleurs par d'autres particularités.

En plus de la situation de la vulve, nous avons dû faire appel également aux caractères donnés par les spicules. Nous avons préféré cependant ne pas suivre exactement la division classique en «spicules égaux et spicules inégaux », car on éloigne ainsi des formes très proches les unes des autres.

Il nous semble qu'en élargissant un peu le cadre des espèces à spicules égaux; on obtienne des groupements plus homogènes. Nous distinguons donc, d'une part, les formes ayant des spicules très 
franchement inégaux, soit par leur taille, soit par leur structure, et, d'autre part, les formes ayant des spicules de structure comparable et de taille égale ou peu différente.

Les six sous-familles que nous obtenons nous paraissent ainsi assez homogènes :

les Cardionematinæ à vulve préanale, définis par Yamaguti (1941) ; les Oswaldofilariinæ n. subfam., parasites de Reptiles à vulve prééquatoriale ;

les Dirofilariinæ à queue courte et à ailes caudales bien développées, tels qu'ils sont définis par Wehr en 1935 ;

les Dipetalonematinæ, qui groupent ici les formes à queue longue et à spicules très franchement inégaux ;

les Splendidofilariinæ n. subfam., dont les spicules sont égaux ou subégaux ;

les Onchocercinæ sont définis ici comme ayant une queue courte et des spicules très différents. Chitwood et Chitwood (1950) avaient déjà repris la sous-famille en s'appuyant sur la dilatation du corps au niveau de la cellule excrétrice. Nous ne croyons pas que cet élément puisse caractériser les Onchocercinæ, car des genres qui paraissent zoologiquement très proches d'Onchocerca (tel que Paronchocerca) en sont dépourvus.

\section{Tableau des familles (1)}

1--(2) Cavité buccale cylindrique bien développée. Tête portant deux pseudo-lèvres latérales.

8 papilles sur le cycle externe et 2 latérales sur le cycle interne. Amphi des très fines. Esophage divisé. Pas d'ailes caudales. Vulve pré- ou postéquatoriale. CEufs entourés d'une membrane vitelline. Souvent une touffe d'épines à la pointe caudale $\ldots \ldots \ldots \ldots \ldots \ldots$. Desmidoceroidæ

2-(1) Cavité buccale réduite ; pas de pseudo-lèvres céphaliques.

3- (4) En plus des papilles, la tête est ornée d'épines.

Queue très courte, pas d'ailes caudales, spicules très inégaux. Vulve antérieure; œufs entourés d'une membrane vitelline, microfilaires circulant dans le sang $\ldots \ldots \ldots \ldots \ldots \ldots \ldots \ldots \ldots$. Stephanofilariidæ

4- (3) Pas d'épines céphaliques en dehors des papilles.

5-(6) Tête nue ou portant des formations cuticulaires variées. CEsophage généralement divisé en une portion antérieure muscu-

(1) Le genre Elaphocephalus Molin 1860 parasite de perroquets, et le genre Solenonema Diesing 1861 parasite de mammifères néotropicaux, sont insuffisamment connus et n'ont pas pu être classés. 
laire et une portion postérieure glandulaire. Ovipares (avec des œufs à coque épaisse) ou vivipares. Premier stade larvaire habituellement court et trapu, rarement long et grêle, l'extrémité antérieure armée de rangée d'épines, l'extrémité postérieure, longue et pointue, ou bien courte et arrondie, et dans ce cas, portant une rangée d'épines ........... Filariidæ

6--(5) Pas de formations cuticulaires céphaliques. Wsophage généralement non divisé. Vivipares, ou rarement ovipares, avec des œufs à coque mince. Premier stade larvaire dn type « micro: filaire », n'étant jamais court et trapu, ses extrémités toujours dépourvues d'épines .............. Dipetalonematidæ

\section{Tableau des genres}

\section{Famille Desmidocercidæ Cram 1927}

Genre unique. Parasites d'Oiseaux .... Desmidocerca Skrjabin 1916 (=Desmidocercella Yorke et Maplestone, 1926).

\section{Famille des Stephanofilariidæ Wehr 1935}

1- (2) Bouche circulaire, surélevée et portant à sa périphérie une couronne de petites épines.

En plus des précédentes, cercle d'épines plus postérieur, interrompu par les amphides, ou 4 à 5 grandes épines dans la région subdorsale. 8 papilles sur le cycle externe. Esophage court, non divisé. Queue du mâle courte, ornée de nombreuses papilles clcacales.

Parasites de Mammifères.Stephanofilaria Ihle et Ihle-Landerberg, 1933.

2-(1) Bouche ovalaire, entourée par quatre petites épines antérieures aux quatres papilles submédianes du cycle externe.

Esophage très long, bien divisé. Queue du mâle très courte, arrondie, dépourvue de papilles cloacales.

Parasites d'Amphibiens ................. Icosiella Seurat, 1917.

\section{Famille des Filariidæ Claus 1885}

\section{Division en sous-familles}

1-(2) Bouche entourée par quatre lèvres coniques.

Tête séparée du corps par une constriction chitinoïde. 8 papilles céphaliques sur le cycle externe et 4 papilles sur le cycle interne. Spicules égaux, ailes caudales absentes. Ovipares. Premier stade larvaire avec des épines sur la tête; la queue, assez longue et fine, étant au contraire inerme. .............. Tetracheilonematina Wehr, 1935. 
2-(1) Bouche dépourvue de lèvres.

3-(4) Tête ornée de formations chitinoïdes latérales en forme de tridents.

Bouche allongée dorso-ventralement. Queue du mâle très courte, sans ailes caudales. Ovipares. Premier stade larvaire portant des épines sur la tête et sur la queuc ............ Diplotriæninæ Skrjabin, 1916.

4-(3) Tête dépourvue de formations chitinoïdes latérales en forme de tridents.

5-(6) Bouche bordée latéralement par des formations chitinoïdes saillantes, ou par une paire d'épaississements chitinoïdes latéraux en forme d'épaulettes, ou présentant à la fois ces deux genres de formations.

Esophage bien divisé. Spicules inégaux, et de structures différentes. Queue du mâle habituellement courte et ailée. Femelles ovipares ou plus rarement vivipares. Premier stade larvaire généralement court et

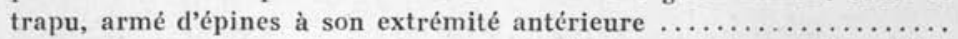
........................... Dicheilonematinæ Wehr, 1935.

6- (5) La tête, dépourvue des formations chitinoïdes latérales précédentes, est nue, ou porte un anneau chitinoïde péribuccal simple, ou encore de fins ornements cuticulaires, qui ne sont pas limités aux aires latérales.

7-(8) Vulve très proche de la bouche.

Bouche circulaire. Spicules inégaux et dissemblables. Ovipares .......

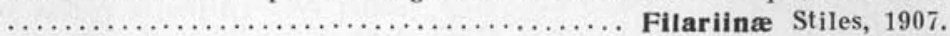

8-(7) Vulve largement éloignée de l'extrémité antérieure.

Bouche circulaire. 8 papilles céphaliques sur le cycle externe. Cycle interne atrophié. Oisophage parfois divisé. Spicules égaux ou peu différents l'un de l'autre. Ovipares. Premier stade larvaire avec des épines

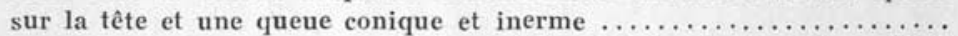
...................... Aproctinæ Yorke et Maplestone, 1926.

Sous-famille des Filariinæ Stiles 1907

Division en genres

1-(2) Cuticule de l'extrémité antérieure couverte de verrucosités allongées transversalement. Anus subterminal.

Parasites de Mammifères ...... Parafilaria Yorke et Maplestone, 1926.

2-(1) Cuticule de l'extrémité antérieure lisse. Queue assez longue.

3-(4) Anneau chitinoïde bien marqué en avant de l'œsophage.

Parasites de Mammifères ............ Hyracofilaria Ortlepp, 1937. 
4-(3) Pas d'anneau chitinoïde bien marqué en avant de l'œsophage. Parasites de Mammifères ................ Filaria Mueller, 1787.

\section{Sous-famille des Dicheilonematinæ Wehr 1935}

\section{Division en genres}

1-(12) Bouche entourée de formations latérales en épaulettes.

2-(7) En plus des formations en épaulettes, la bouche est bordée latéralement par des éminences chitinoïdes saillantes en avant.

3-(4) Spicule gauche relativement court et effilé, muni d'une aile et fortement strié dans sa partie distale.

Parasites d'Oiseaux ............... Dicheilomena Diesing, 1861. (= Contortospiculum Skrjabin, 1915).

4-(3) Spicule gauche fin, très long, et non strié dans sa partie distale.

5-(6) Ailes spiculaires bien développées, et largement fusionnées postérieurement. Ailes caudales soustendues par de grandes papilles cloacales.

Parasites de Reptiles ............. Hastospiculum Skrjabin, 1923. (= Setarospiculum Mirza et Basir, 1939).

6-(5) Ailes spiculaires très étroites, papilles cloacales séparées des ailes caudales (excepté chez $H$. pertenuialatum).

Parasites d'Oiseaux ............. Hamatospiculum Skrjabin, 1916 (=Parhamatospiculum Skrjabin et Petrov, 1928).

7- (2) Formations en épaulettes simples, dépourvues d'éminences chitinoïdes latérales saillantes en avant.

8-(11) Formations en épaulettes bien marquées. Queue du mâle très courte, ailée. Esophage très fortement divisé.

9 - (10) Formations en épaulettes directement en contact avec la bouche, spicules trapus, très largement ailés et fortement striés. Ovipares.

Parasites d'Oiseaux ............. Serratospiculum Skrjabin, 1915.

10- (9) Formations en épaulettes séparées de la bouche par un très large anneau. Spicules très inégaux : le gauche, fin, très long, et sans ailes. Vivipares.

Parasites d'Oiseaux ................ Heterospiculum Schigin, 1951.

11- (8) Formations en épaulettes légères, en forme d'étoiles à six pointes. Queue du mâle assez longue, œsophage court et non divisé. Vivipares.

Parasite cardiaque de Phoques ........ Skrjabinaria Lubimov, 1927. 
12-(1) Bouche dépourvue de formations latérales en épaulettes.

13-(18) Bouche bordée par des dents latérales saillantes en avant.

14 - (15) Autour de la bouche, en plus des dents latérales, présence d'une dent ventrale et d'une dent dorsale. Queue de la femelle longue, pourvue d'une boule plus ou moins épineuse. Vivipares.

Parasites de Mammifères ................. Setaria Viborg, 1795.

15-(14) Pas de dent ventrale ni dorsale autour de la bouche. Queue très courte.

16-(17) Dents latérales simples. Pas de capsule buccale. Queue du mâle ailée. Ovipares.

Parasites d'Oiseaux ................Monopetalonem 2 Diesing, 1861. (=Politospiculum Skrjabin, 1916 ; = Ornithosetaria Sandground, 1933).

17-(16) Dents latérales trifurquées. Capsule buccale très forte. Queue du mâle sans ailes. Vivipares.

Parasites d'Oiseaux ............. Pharyngosetaria Lubimov, 1937.

18-(13) Ornementations péribuccales ne comportant pas de dents latérales saillantes en avant.

19-(20) Bouche ornée latéralement par une paire de petits anneaux chitinoïdes posés sur la cuticule. Queue de la femelle tronquée avec de nombreux petits tubercules apicaux. Ovipares.

Parasites de Mammifères .............. Suifilaria Ortlepp, 1937.

20-(19) Bouche portant seulement deux épaississements latéraux plats. Queue de la femelle digitiforme, avec deux épaississements latéraux à l'extrémité. Vivipares.

Parasites de Mammifères ........... Papillosetaria Vevers, 1922.

\section{Sous-famille des Diplotriaeninæ Skrjabin 1916}

\section{Division en genres}

1- (2) Quatre pièces chitinoïdes digitiformes, appliquées latéralement contre l'extrémité antérieure de l'œsophage.

Parasites d'Oiseaux ............... Quadriplotriaena Wehr, 1939.

2-(1) Deux pièces chitinoïdes en forme de trident, appliquées latéralement contre l'extrémité antérieure de l'œsophage.

3-(4) Pièces chitinoïdes grandes et fortement cuticularisées.

Parasites d'Oiseaux ......... Diplotriaena Railliet et Henry, 1909.

4- (3) Pièces chitinoïdes petites et faiblement cuticularisées.

Parasites d'Oiseaux .............. Diplotriaenö̈des Walton, $192 \pi$. 
Sous-famille des Aproctinæ Yorke et Malplestone 1926

Division en genres *

1-(6) Capsule buccale relativement grande, formée par un anneau chitinoïde bien marqué.

2-(3) Capsule buccale formée par un anneau chitinoïde très épais, faisant saillie en avant de la tête. Esophage divisé. Spicules égaux. OEufs à coque épaisse.

Parasites d'Oiseaux ........ Austrofilaria Johnston et Mawson, 1940.

3- (2) Capsule buccale formée par un anneau chitinoïde plus faible, non saillant, en avant de la tête. OEsophage non divisé. Cuticule du pourtour buccal, en général épaissie, et plus ou moins festonnée.

4-(5) Cuticule ornée de petites papilles. Capsule buccale séparée de l'œsophage. Spicules subégaux.

Parasites d'Oiseaux ............. Squamofilaria Schermling, 1925. (=Eucamptes Duj., $1845 ;$ = Coronofilaria Yorke et Maplestone, 1926).

5- (4) Cuticule lisse. Capsule buccale incluse dans le tissu œsophagien. Mâle inconnu.

Parasite du tissu nerveux de Mammifères. Neurofilaria Whitlock, 1952.

6-(1) Capsule buccale petite ou absente.

7-(8) Présence de fins cordons, lobés entre papilles et amphides, sur la cuticule céphalique. Cavité buccale petite. Esophage court et simple. Spicules égaux. Wufs à coque épaisse.

Parasites d'Oiseaux ............. Pseudaprocta Schikhobalowa, 1930.

8- (7) Pas de cordons lobés entre papilles et amphides sur la cuticule céphalique.

9 -(14) Queue du mâle ornée d'ailes et pourvue de nombreuses papilles.

Esc.phage simple. Spicules égaux.

10 -(13) Queue du mâle largement ailée et pourvue de grosses papilles. Capsule buccale absente. Vivipares.

11-(12) Ailes latérales étendues sur toute la longueur du corps. Queue du mâle asymétrique.

Parasites d'Oiseaux. Spirofilaria Yamaguti, 1935, emend. Baylis, 1944.

$\left(^{*}\right)$ Nous rappelons que les caractères larvaires de certains genres sont insuffisamment connus, et, que dans ces cas, il devient impossible de les placer avec certitude parmi les Filariidæe ou parmi les Dipetalonematidx. Les sous-familles des Splendidofilariina, et des Aproctina contiennent l'une et l'autre des formes très spécialisées. Le diagnostic, en l'absence des caractères larvaires, devient spécialement difficile, et certains genres sont donc placés ici de façon provisoire. 
12-(11) Ailes latérales n'intéressant pas toute la longueur du corps. Queue du mâle symétrique.

Parasites d'Oiseaux ............. Pelicitus Railliet et Henry, 1910.

(=Eulimdana Founikoff, 1934).

13-(10) Queue du mâle portant de petites ailes et de nombreuses petites papilles. Capsule buccale petite. Eufs à coque mince.

Parasites d'Oiseaux ................. Buckleyfilaria Singh, 1949.

14- (9) Queue du mâle dépourvue d'ailes.

15-(16) Bouche bordée par deux petites lèvres. OEsophage divisé.

Anus distinct. Ovipares. Parasites d'Oiseaux. Lissonema Linstow, 1903.

16-(15) Bouche simple sans lèvres. Esophage non divisé.

17-(18) Pointe caudale lisse. Spicules égaux.

Parasites des cavités oculaires ou nasales des Oiseaux $\ldots \ldots \ldots \ldots \ldots$

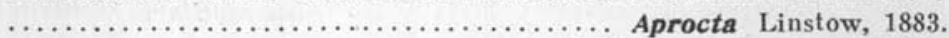

(= Striatofilaria Lubimov, 1927).

18-(17) Pointe caudale pourvue de deux digitations bien développées dans les deux sexes. Une seule papille précloacale. Spicules légèrement inégaux.

Parasites sous-cutanés des Oiseaux ...... Ularofilaria Lubimov, 1946.

Sous-famille des Tetracheilonematinæ Wehr 1935

Genre unique. Parasite d'Oiseaux.... Tetracheilonema Diesing, 1861. (= Labiatofilaria Adams, 1933).

Famille des Dipetalonematidæ Wehr 1935

Division en sous-familles

1-(4) Vulve postérieure ou moyenne.

2-(3) Vulve préanale.

Parasites de Mammifères ....... Cardionematinæ Yamaguti, 1941.

3-(2) Vulve s'ouvrant dans la partie moyenne du corps.

Parasites de Reptiles ............ Oswaldofilarinæ n. subfam.

4-(1) Vulve antérieure.

5-(6) Ailes caudales du mâle bien développées. Esophage généralement divisé en deux parties. Queue généralement courte .... $\ldots \ldots \ldots \ldots \ldots \ldots \ldots \ldots \ldots \ldots$ Dirofilariinæ Wehr 1935. 
6- (5) Pas d'ailes caudales bien développées (à l'exception de quelques espèces d'Onchocerca).

7-(8) Nématodes ayant à la fois une queue longue (1) et des spicules très différents l'un de l'autre .. Dipetalonematinæ Wehr 1935.

8- (7) Nématodes n'ayant pas à la fois une queue longue et des spicules très différents l'un de l'autre.

9 -(10) Queue longue ou courte et spicules égaux ou peu différents l'un de l'autre ............... Splendidofilariinæ n. subfam.

10-(9) Queue courte, et spicules très différents l'un de l'autre ...... $\ldots \ldots \ldots \ldots \ldots \ldots \ldots \ldots \ldots \ldots \ldots$ Onchocercinæ Leiper 1911.

\section{Sous-famille des Cardionematinæ Yamaguti 1941}

Genre unique : bouche sans lèvres ni capsule buccale. Esophage court, non divisé. CEufs à coque mince.

Parasite du cœur de Mammifères ....... Cardionema Yamaguti, 1941

\section{Sous-famille des Oswaldofilariinæ n. subfam.}

\section{Division en genres}

1-(4) Spicules égaux. Vulve équatoriale.

2- (3) Pas de capsule buccale. Spicules épais et très courts. Queue de la femelle ornée de grosses papilles subventrales.

Parasites de Reptiles.... Conispiculum Pandit, Pandit et Iyer, 1929.

3- (2) Capsule buccale de petite taille. Queue de la femelle lisse.

Parasites de Reptiles ............. Piratuba Freitas et Lent, 1941.

4-(1) Spicules inégaux. Vulve dans la moitié antérieure du corps.

Parasites de Reptiles ............ Oswaldofilaria Travassos, 1933.

\section{Sous-famille des Dirofilariinæ Wehr 1935}

Division en genres

1-(4) Cuticule ornée.

2-(3) Cuticule ornée de bâtonnets longitudinaux. Queue relativement longue.

Parasites d'Edentés .......... Bostrichodera Sandground, 1938 (2).

(1) Nous entendons par espèces à « queue longue », les formes où la longueur anus-pointe caudale est supérieure au double du diamètre du corps au niveau de l'anus.

(2) Le genre Skrjabinodera Gnedina et Vsevolodov, 1947, dont nous n'avons pas pu avoir la description originale, est signalé comme proche des Bostrichodera. 
$3 \div$ (2) Cuticule ornée de bosselures. Queue courte.

Parasites de Primates ....................... Loa Stiles, 1905.

(= Paraloa Rodhain et Van den Berghe, 1939).

4-(1) Cuticule lisse.

5-(6) Anneau chitineux bien marqué en avant de l'œsophage.

Queue courte et arrondie.

Parasites de Primates ................. Tawila Khalil, 1932.

6- (5) Pas d'anneau chitineux bien marqué en avant de l'œsophage.

7-(8) Amphidelphie. Parasites d'animaux à sang froid.

Parasites de Reptiles et d'Amphibiens........ Foleyella Seurat, 1917.

$(=$ Folegellides Caballero, 1935).

8-(7) Opistodelphie. Parasites de Mammifères.

9 -10) Esophage non divisé. Queue du mâle pointue.

Parasites de Rongeurs .......... Dirofilariaeforma Lubimov, 1935.

10-(9) Esophage divisé. Queue du mâle obtuse.

Parasites de Mammifères........ Dirofilaria Railliet et Henry, 1911.

Sous-famille des Dipetalonematinæ Wehr 1935

Division en genres

1-(2) Queue du mâle portant une callosité cuticulaire ventrale, subterminale.

Cavitẻ buccale chitinisée. Gisophage simple. Femelle inconnue.

Parasites d'Oiseaux ............... Hamulofilaria Chandler, 1924.

2-(1) Queue du mâle dépourvue de callosité cuticulaire.

3 -(12) Capsule buccale bien marquée.

4-(5) Ovéjecteur hypertrophié. Ailes caudales du mâle relativement bien développées. Capsule buccale courte, étranglée en son milieu, saillante en avant de la tête.

Parasites de Marsupiaux ......... Skrjabinofilaria Travassos, 1925.

(= Cortiamosoides Foster, 1939).

5-(4) Nématodes n'ayant pas tous ces caractères à la fois.

6-(9) Cavité buccale mince, tubuliforme, très allongée.

7-(8) Petites ailes caudales chez le mâle. Queue de la femelle tétrapétalée.

Parasites de Primates ................ Parlitomosa Naghati, 1935. 
8-(7) Pas d'ailes ni de papilles chez le mâle. Queue de la femelle avec seulement deux très petites pointes.

Parasites de Rongeurs ........... Pseudolitomosa Yamaguti, 1941.

9-(6) Cavité buccale plus courte et plus épaisse que précédemment.

10-(11) Capsule buccale cylindrique. Essophage en bulbe antérieurement. Papilles postcloacales présentes. Pointe caudale inerme dans les deux sexes.

Parasites de Mammifères ............Litomosoüdes Chandler, 1931.

(=Vestibulosetaria Vogel et Gabaldon, 1932 ; = Finlaynema Vigueras, 1934).

11-(10) Capsule buccale courte et conique. Pas de papilles postcloacales. Queue de la femelle généralement armée de petites pointes.

Parasites de Chiroptères........ Litomosa Yorke et Maplestone, 1926.

12-(3) Capsule absente, ou réduite à un anneau accolé à l'extrémité antérieure de l'œsophage.

13-(20) Pointe caudale lisse dans les deux sexes.

14-(15) Tête très dilatée. Queue brusquement tronquée dans les deux sexes.

Parasites de Chiroptères .......... Migonella Lent et Freitas, 1946.

15-(14) Tête peu ou pas dilatée. Queue arrondie.

16-(19) Anneau chitinoïde accolé à l'extrémité antérieure de l'œsophage.

17-(18) Queue du mâle dépourvue de papilles.

Parasites de Rongeurs ...........................

18-(17) Papilles cloacales du mâle bien développées.

Parasites de Reptiles ............. Macdonaldius Khanna, 1933 (1).

19-(16) Pas d'anneau chitinoïde à l'extrémité antérieure de l'œsophage.

Corps rétréci en arrière de la tète pour former un cou. Gubernaculum bien développé.

Parasites de Primates ............ Wuchereria Silva Araujo, 1877.

20-(13) Pointe caudale ornée de petits appendices coniques ou en forme de languettes.

21-(22) Tête pourvue de deux lèvres latérales.

Parasites de Mammifères .......... Molinema Freitas et Lent, 1943.

(1) Le genre Bhalfilaria Bhalerao et Rao, 1944, parasite dans le cœur des Oiseaux, serait proche de Macdonaldius (Helm. Abst.), mais le mâle est inconnu. 
22 -(21) Pas de lèvres latérales.

23-(24) Grand spicule divisé en deux portions : une proximale cylindrique, et une distale aplatie et souvent membraneuse.

Parasites de Mammifères .......... Dipetalonema Diesing, 1861 (1). (=Acanthocheilonema Cobbold, 1870 ; = Tetrapetalonema Faust, 1935 ; = Loxodontofilaria Van den Berghe et Gillain, 1939; = Mönnigofilaria Skrjabin et Schikhobalowa, 1948) (2).

24-(23) Grand spicule non divisé en deux portions.

Parasites de Marsupiaux........ Breinlia Yorke et Maplestone, 1926.

\section{Sous-famille des Onchocercinæ Leiper 1911}

1- (2) Parasites d'animaux à sang froid.

Plaque cuticulaire chitinoïde préanale.

Farasite de Batraciens ............. Ochoterenella Caballero, 1944.

2-(1) Parasites d'animaux à sang chaud.

3 -(10) Parasites d'Oiseaux.

4-(5) Esophage non divisé. Petit anneau chitinoïde en avant de l'œsophage.

Mâle inconnu.

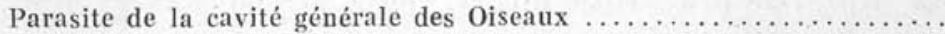
.................... Paralemdana Johnston et Mawson, 1940.

5- (4) Esophage divisé. Pas d'anneau chitinoïde en avant de l'œsophage.

6- (7) Partie distale du grand spicule membraneuse.

Cuticule ornée. Diérides très visibles.

Parasites des vaisseaux des Oiseaux ........................ Alcefilaria Oschmarin et Belouss, 1951.

7-(6) Grand spicule de structure homogène.

8- (9) Grand spicule une ou deux fois plus long que le petit. Cuticule ornée.

Parasites cardiaques des Oiseaux........ Paronchocerca Peters, 1936. (= Houdemerus Chow, 1939 ; = Chinesocerca Skrjabin et Schikhobalowa, 1937).

(1) Le genre Mansonella Faust, 1926, parasite de Primates, es! trop mal connu pour qu'une diagnose soit possible. Il semble pouvoir être placé provisoirement près des Dipetalonema.

(2) Filaria roemeri Linst., 1905 , nous paraît finalement devoir être rattachéc aux Dirofilaria. Filaria evansi Lewis, 1882, qui a également une queue courte, est tellement différente des Dipetalonema, que nous avons cru utile de créer pour elle un genre nouveau parmi les Onchocercina. 
9-(8) Grand spicule au moins 3,5 fois plus long que le petit. Cuticule lisse.

Parasites de la cavité générale des Oiseaux. Lemdana Seurat, 1917 (1).

10- (3) Parasites de Mammifères.

11-(12) Vulve très antérieure. Les six papilles céphaliques du cycle interne bien développées.

Parasites dans les tumeurs fibreuses de Mammifères

Pseudofilaria Sandground, 1935).

(= Protofilaria Sandground, 1935).

12-(11) Vulve éloignée de la bouche. Papilles céphaliques du cycle interne atrophiées ou réduites à deux latérales.

13-(14) Tête aplatie et élargie latéralement. Face ventrale de la queue de la femelle, portant un gros bourrelet charnu.

Parasite des vaisseaux des Mammifères

....................... Deraïophoronema Romanovitch, 1916.

[Espèce type : D. evansi (Lewis, 1882) (2)].

14-(13) Tête arrondie. Pas de bourrelet charnu sur la queue de la femelle.

15-(18) Anus de la femelle terminal ou subterminal. Esophage divisé.

16-(17) Extrémité antérieure du corps très atténuée. Papilles cloacales sessiles. Vivipare.

Parasite cardiaque des Ruminants....... Cordophilus Mönnig, 1926.

17-(16) Extrémitẻ antérieure du corps normale. Papilles cloacales grosses et pédonculées.

Ovéjecteur pourvu d'un réceptacle sacciforme. Ovipare.

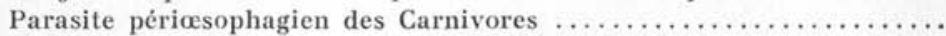

................... Cystofilaria Skrjabin et Schikhobalowa, 1948.

18-(15) Anus de la femelle distant de la pointe caudale. Esophage non divisé.

19-(20) Quadridelphie.

Esophage très long. Corps de la femelle beaucoup plus large en arrière qu'en avant.

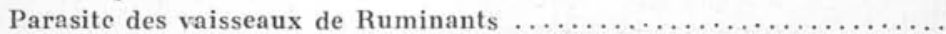

.......................... Elaeophora Railliet et Henry, 1912.

(1) Nous croyons pouvoir placer l'espèce L. micropenis Travassos, 1925, dans le genre Aproctiana.

(2) Nous reprenons ici le nom « Deraïophoronema cameli » Romanovith, 1916, précisément synonyme de l'espèce que nous proposons de séparer du genre Dipetalonema. 
20-(19) Didelphie.

21-(22) Cuticule rugueuse. Papilles groupées autour du cloaque et de la pointe caudale.

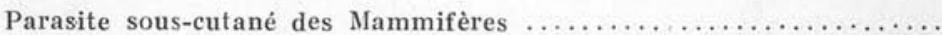
.......................Wehrdikmansia Caballero, 1945. (= Acanthospiculum Skrjabin et Schikhobalowa, 1948).

22-(21) Cuticule pourvue d’annulations. Papilles pré-cloacales présentes.

Parasites des vaisseaux et des tissus de Mammifères . . . . . . . . . . .

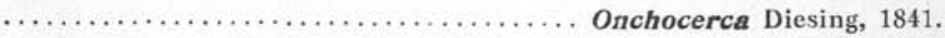

Sous-famille des Splendidofilariinæ nov. subfam.

\section{Division en genres}

1-(20) Queue longue ou courte. Anus de la femelle rarement atrophié et nettement distant de la pointe caudale.

2- (5) Parasites de Reptiles.

3-(4) Queue du mâle longue, sans ailes ni papilles. Amphidelphie.

Parasites de Lézards .............. Thamugadia Seurat, 1917.

4-(3) Queue du mâle courte, avec de petites papilles. Opistodelphie. Parasites du cœur des Tortues ............. Cardianema Alicata, 1933.

5- (2) Parasites d'Oiseaux et de Mammifères.

6-(17) Parasites d'Oiseaux.

7-(8) Extrémité antérieure portant deux ailes cervicales latérales.

Spicules subégaux, très petits.

Parasite cardiaque d'Oiseaux .......... Lerouxinema Singh, 1949.

8-(7) Extrémité antérieure dépourvue d'ailes cervicales latérales.

9-(10) Queue longue avec papilles cloacales bien développées.

Parasites sous-cutanés ou cardiaques des Oiseaux

Splendidofilaria Skrjabin, 1923.

(= Chandlerella Yorke et Maplestone, 1926, p.p. ; = Vagrifilaria Augustine, 1937, p.p. : = Parachandlerella Caballero, 1948) (1).

(1) Singh (1949) a attiré l'attention sur le fait que les bosses cuticulaires qui servaient à caractériser le genre Splendidofilaria ne sont souvent distinguables que sur les spécimens vivants et que les genres Splendidofilaria et Chandlerella sont synonymes. Ces caractères cuticulaires avaient d'ailleurs amené un groupement des espèces très hétérogènes dans le genre Splendidofilaria et dans les gen- 
10 - (9) Queue courte.

11-(12) Papilles cloacales bien développées.

Parasites d'Oiseaux ................ Paramicipsella Chow, 1939. $(=$ Chandlerella Yorke el Maplestone, 1926, p.p. ; = Vagrifilaria Augustine, 1937, p.p.) (1).

12-(11) Papilles cloacales absentes ou plates et peu nombreuses.

13-(14) Vulve au niveau de l'œsophage. Vagin très court, donnant tout de suite deux utérus.

Parasites des Oiseaux ............. Ornithofilaria Gönnert, 1937.

14 -(13) Vulve postœsophagienne. Vagin long.

15-(16) Spicules égaux et très petits.

Parasites des Oiseaux .............. Carinema Pereira et Vaz, 1925.

16-(15) Spicules nettement inégaux, l'un presque le double de l'autre.

Parasites des Oiseaux ............. Paraprocta Maplestone, 1931.

17-(6) Parasites de Mammifères.

18 - (19) Bosses cuticulaires sur les aires latérales et sur l'extrémité postérieure du mâle. Papilles cloacales distribuées régulièrement le long de la queue.

Parasites des Rongeurs .................. Micipsella Seurat, 1921. (= Cercofilaria Kalantanian, 1924).

19 - (18) Cuticule portant des épaississements fusiformes distribués irrégulièrement. Papilles groupées autour du cloaque et sur la pointe caudale du mâle.

Parasites de Mammifères... Onchocercella Yorke et Mapplestone, 1931. $(=$ Grammophora Gedoelst, 1916; = Katanga Yorke et Maplestone, 1926).

20-(1) Queue très courte. Anus de la femelle terminal, subterminal ou atrophié.

res voisins. En nous basant sur la longueur de la queue, nous eroyons pouvoir séparer deux groupes :

$1^{\circ}$ Les Splendidofilaria (à queue longue) avec pour espèce-type $S$. páblonvsligi Skrjabin, 1923, où l'on peut ranger : $S$. bosei (Chandler, 1924), n. comb.; $S$. gedoelsti Travassos, $1925 ; S$. sinensis (Li, 1933), n. comb.: $S$. columbigallina (Augustine, 1937), n. comb. et $S$. periarterialis (Caballero, 1948), n. comb.

$2^{\circ}$ Les Paramicipsella (à queue courte) avec pour espèce-type $P$. brevicaudata Chow, 1939, où l'on peut ranger : $P$. gedoelsti (Travassos, 1925), n. comb.; $P$. stantchinsky (Gilbert, 1932), n. comb.; P. lepidogrammi (Tubangui et Masilungan, 1937), n. comb. ; P. travassosi (Koroliowa, 1926), n. comb. ; P. lienalis (Orloff, 1947), n. comb. ; P. australis (Johnston et Mawson, 1942), n. comb. et P. brevispiculum (Singh, 1949), n. comb. 
21-(26) Esophage nettement divisé en deux portions.

22-(23) Cloaque du mâle s'ouvrant presque à la pointe caudale.

Parasites de Reptiles ............................. 1924.

23-(22) Cloaque du mâle nettement distant de la pointe caudale.

24-(25) Esophage court. Intestin présent. Cuticule lisse.

Parasites des cavités orbitaires des Oiseaux Aproctoïdes Chandler, 1929.

25-(24) Esophage très long. Intestin réduit à un petit appendice à la fin de l'œsophage. Cuticule annelée.

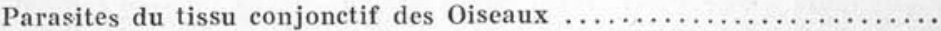

............................ Anenteronema Oshmarin, 1949.

26-(21) Esophage non divisé.

27-(28) Cuticule avec annulations transverses. Queue du mâle aplatie dorso-ventralement.

Parasites du muscle cardiaque des Oiseaux... Sarconema Wehr, 1939.

28-(27) Nématodes n'ayant pas ces caractères.

29-(30) Esophage très court et transparent. Queue arrondie courte et massive dans les deux sexes. Ovipares.

Parasites des Oiseaux .................. Eufilaria Seurat, 1921.

30 -(29) Nématodes n'ayant pas ces caractères.

31-(32) Queue de la femelle scindée à l'apex par une fente dorso-ventrale. Parasites de Mammifères.

Parasites de Lémuriens ............ Protofilaria Chandler, 1929.

32-(31) Queue de la femelle arrondie. Parasites des Oiseaux.

33-(34) Cloaque subterminal. Capsule buccale cylindrique bien distincte ou absente.

Parasite abdominal des Oiseaux..........Aproctiana Skrjabin, 1934.

34-(33) Cloaque distant de la pointe caudale. Capsule buccale très courte ou absente.

35-(36) Cuticule divisée en quatre champs, les deux médians avec fortes striations longitudinales, les deux latéraux lisses.

Parasite abdominal des Oiseaux........... Aproctella Cram, 1931.

36-(35) Cuticule non divisée en quatre champs différents.

37--(38) Queue du mâle avec nombreuses papilles postcloacales. Vivipare.

Parasite de la cavité orbitaire des Oiseaux Skrjabinocta Chertkova, 1946. 
38-(37) Queue du mâle plus courte, sans papilles: Ovipares.

Parasite du péricarde des Oiseaux........ Cardiofilaria Strom, 1937.

\section{RÉSUMÉ}

Pour permettre la détermination générique des Filaires, les tableaux dichotomiques de Yorke et Maplestone sont beaucoup trop anciens. Ceux de Skrjabin et de Schikhobalova sont rédigés en russe, dans un livre difficile à se procurer, et sont basés sur des caractères qui semblent trop artificiels. Au contraire, les coupures en familles et sous-familles, proposées par Wehr, sont très satisfaisantes, car elles paraissent correspondre aux processus évolutifs subis par les Spirurides qui s'adaptent à la vie tissulaire. Ces divisions sont donc beaucoup moins artificielles, et permettent effectivement des groupements relativement homogènes.

Il nous a donc semblé utile de donner de nouveaux tableaux dichotomiques en utilisant le cadre général publié par Wehr. Nous conservons toutes les familles et sous-familles proposées par cet auteur, à l'exception des Dipetalonematidæ, qui sont scindés en six sousfamilles : les Cardionematinæ, les Oswaldofilariinæ, les Dirofilarii$n æ$, les Dipetalonematinæ, les Splendidofilariinæ, et les Onchocercinæ. Pour des raisons d'ordre phylogénique et pratique, ces divisions sont généralement basées sur la longueur de la queue.

Nous admettons 89 genres valides ; 30 genres sont placés en synonymie ; le genre Deraïophoronema est repris pour placer la Filaria evansi (Lewis 1882). Le grouve des espèces placées précédemment dans les genres Splendidofilaria, Chandlerella, Parachandlerella, Vagrifilaria et Paramicipsella est remanié, et les espèces sont réparties simplement entre les deux genres : Splendidofilaria et Paramicipsella.

\section{Bibliographie}

Caballero (y C. E.). - Estudios helminthologicos de la region oncocercosa de Mexico y de la Republica de Guatemala, Nematoda, 6a. Parte. Y algunas consideracions en torno a los generos Onchocerca Diesing, 1841, y Acanthospiculum Skrjabin y Schikobalowa, 1948, Ann. Inst. de Biol., XXII, 1951, 141-158, fig. $1-3+1$ pl.

Chitwoon (B. G.) et Chitwood (M. B). - An introduction to Nematology, Section 1. Anatomy, viII + 213 pp., fig. 1-145, Baltimore, 1950.

Singh (S. N.). - Studies on the Helminth Parasites of Birds in Hyderabad State. Nematoda IV. Journ. of Helminth., XXIII, 1949, 39-56, fig. 1-32. 
Sкrjabin (K. J.) et Schiкhobalova (N. P.). - Contribution au remaniement de la classification des Nématodes de l'ordre des Filariata Skrjabin, 1915. Ann. Parasit., XIV, 1936, 61-75.

SкRJabin (K. J.) et Schiкhoвalova (N. P.). - Filaires animales et humaines, 608 pp., fig. 1-256, Moscon, 1948. (En russe).

Wenr (E. E.). - A revised classification of the Nematode superfamily Filarioidea. Proc. Helm. Soc. Wash., II, 84-88, 1935.

Yorke (W.) et MAPLestone (P. A.). - The Nematodes parasites of Vertebrates, $\mathrm{x}+536$ pp., fig. 1-307, Londres, 1926 .

(Laboratoire de parasitologie de la Faculté de médecine de Paris Directeur : H. Galliard) 